\title{
Improvement in reliability of coverage using 2-hop relaying in cellular networks
}

\author{
Ansuya Negi \\ Department of Computer Science \\ Portland State University \\ Portland, OR, USA \\ negi@cs.pdx.edu
}

\author{
Suresh Singh \\ Department of Computer Science \\ Portland State University \\ Portland, OR, USA \\ singh@cs.pdx.edu
}

\begin{abstract}
It has been shown that gaps exist in the useful coverage service area of a cell and the reliability of coverage drops due to buildings as well as interference in CDMA cells. We have used the Generalized Poisson Process to model the areas where signal level fall below threshold and formulated the mathematical definition of useful service area in the presence of relays. We used approaches "with replacement" and "without replacement" of idle mobiles to serve as relays or intermediaries. We also use a constraint to make sure that the intermediary doesn't fall in a below threshold area. Monte Carlo simulations show that 2-hop relaying can increase the useful service coverage area by $4-14 \%$ in sparse and heavy building density areas.
\end{abstract}

Keywords-reliable coverage, useful service area, CDMA, relaying

\section{INTRODUCTION}

Coverage is defined as the farthest distance from the base station where the signal has reliable signal strength [1], which is also the range of the cell. There is, however, the question of how uniform this coverage is. Using accurate ray tracing models, Coinchon et al. [2] report $12 \%$ rejections when $20 \mathrm{~dB}$ building penetration loss is not taken into account and $20 \%$ rejections when the building penetration loss is taken into account. Intuitively, the reason is that path loss contours in high building density areas make their own local minima. Lee [3] terms these as pockets in which the propagation can be very poor. Lee also shows that, with the CCIR propagation model, the impact of the building density on the cell radius is much more than the antenna heights. Further evidence is provided by Neskovic et al. [4], where they reported coverage in a city. Areas identified as highly dense typically have lower signal strength when compared to semi-urban areas.

Cellular planners identify such areas as Below Threshold Areas (BTA). Their solution is to add extra base station or an additional sector if the degradation is extreme. In general, the mobiles located in BTAs increase their power to overcome the rejections. But in CDMA type interference limited systems, increasing power increases the interference in the cell, reducing the capacity of the system [5]. This is a tradeoff, as coverage has an inverse relationship with capacity of the cell. Jones and Skellern [9] report that contiguous cell coverage may not be possible in a microcell even if the call loss rate is low. It analyzes interference to noise ratio and shows that interference from other mobiles is sufficient to produce gaps in the coverage.
In this paper we use the metric "useful service area" [1] to identify the amount of area where the signal level is above some threshold. It can thus be seen as a measure of the reliability of coverage. This measure is quite relevant now as more and more people convert to wireless and expect good coverage.

We have introduced next generation stretchable architecture in our earlier paper [6]. In this architecture, a mobile's call can be stretched by making an intermediary carry its call, resulting in a 2-hop call. The intermediary can be another mobile or a car with sufficient power resources. In the simulations given in [6] the choice of the intermediary was influenced by whether we can save energy by carrying the call. We recognize the potential of the stretchable architecture to solve the above service coverage area problem. If a mobile is stuck in some below threshold area, it need not increase its power to reach the base station. It can instead search for another idle mobile, which can relay its call to the base station. This procedure is particularly useful in a dead spot [7], as the base station may be out of reach for the mobile. Thus, in addition to saving battery resources, since a low signal power is required to reach the relay, we can increase coverage.

This model, however, may increase the intra-cell interference. Since we don't know which mobile will be selected as a carrier we can't predict the increase in interference. An analysis of possible capacity increase [8] shows a $20 \%$ increase in pole capacity for uplink and negligible capacity gain in downlink due to loss of synchronized transmission.

In this paper we analyze the path loss contours in Section II to identify below threshold areas. We introduce a model to predict the occurrences of these areas using the Generalized Poisson Distribution model. We also formulate the stretchable call assisted useful coverage area problem. In Section III we report the results of Monte Carlo simulations to show the increase in useful coverage area using the stretched model. Finally we conclude in Section IV.

\section{MATHEMATICAL ANALYSIS}

To show the impact of building density we plot path loss contours for $850 \mathrm{MHz}$ for a typical urban area. The area of the cell is $1 \mathrm{~km}^{2}$. The base station antenna height is $30 \mathrm{~m}$ and mobile antenna height is $1.5 \mathrm{~m}$.

This work was funded by the NSF under grant ANIR - 9980485, 0196042. 


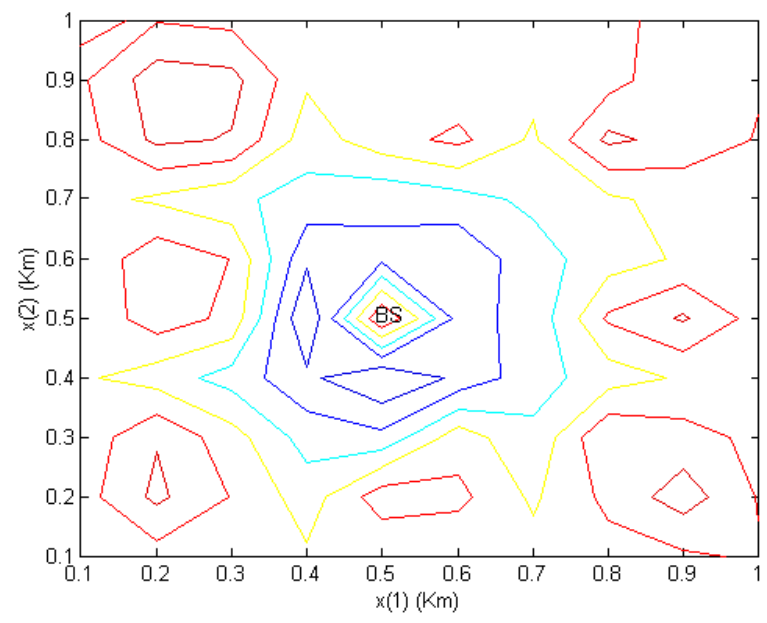

Figure 1. Path Loss Contours in $\mathrm{dB}$, showing local minima at buildings

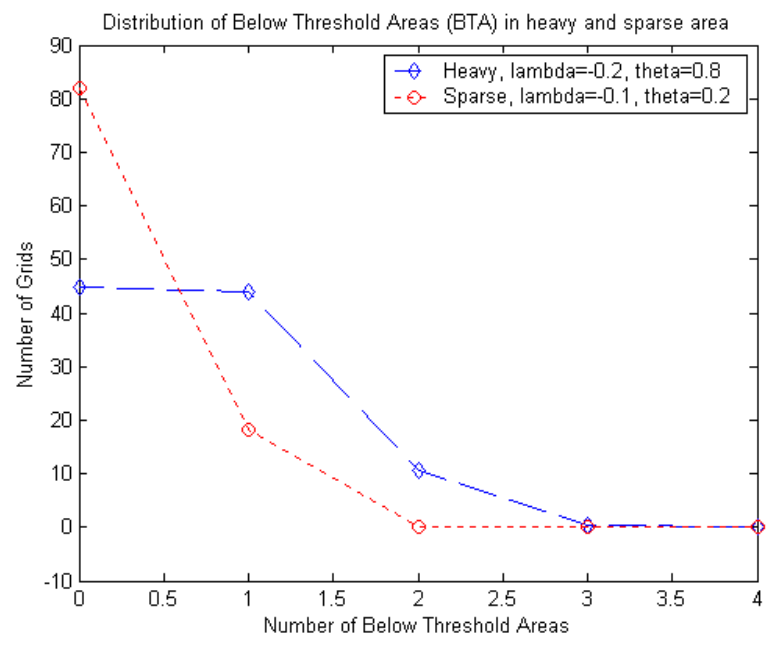

Figure 2. Distribution of below threshold areas using generalized poisson distribution

Figure 1 plotted using CCIR formula [3] illustrates how high building density introduces local gaps in the contours. The base station is at the center of the cell. Only the area near the base station shows the circular curves as expected. The contours get distorted as we go further away from the base station due to the presence of buildings. These areas may fall below the threshold resulting in loss of calls.

In order to predict the occurrences of areas falling below the threshold signal, we discretize the cellular space into grids. As shown in Figure 1, the signal distorts around buildings, we therefore use high building density areas as the candidates having more Below Threshold Areas (BTA). To properly capture this clustering of areas we use Generalized Poisson Distribution (GPD) [10]. It is a two parameter model given as,

$$
\begin{aligned}
& P_{X}(\theta, \lambda)=\frac{\theta(\theta+x \cdot \lambda)^{x-1} e^{-\theta-x \cdot \lambda}}{x}, x=0,1,2, \ldots \\
& P_{X}(\theta, \lambda)=0, x>m, \text { when } \lambda<0
\end{aligned}
$$

where $\theta>0, \max (-1, \theta / \mathrm{m})<\lambda<=1$ and $\mathrm{m}(>=4)$ is the largest positive integer for which $\theta+\lambda / \mathrm{m}>0$ when $\lambda$ is negative.

We use the metric useful service area (or useful coverage area) as the probability that signal strength is above some threshold level, given as

$$
\int_{\text {area }, A} \operatorname{Pr}(\xi>\gamma) d A
$$

where, $\xi$ is the signal level and $\gamma$ is some threshold level. This gives the probability that a connection will not be lost. This covers the entire cellular area where the signal lies above the threshold.

Improvement in the useful coverage area due to stretched call model is given by,

$$
\left.\int_{\text {area }, A} \operatorname{Pr}(\xi\rangle \gamma\right) d A+\int_{\text {area }, A} \operatorname{Pr}(\xi\langle\gamma) * \delta(\text { stretch }) d A
$$

where, $\delta$ (stretch) gives the possibility of a stretched call wherever possible. If the delta function is one, we add to the useful coverage area. The probability that the above delta function will be unity is equal to the probability of a calling mobile in the BTA finding another mobile to relay its call. The relaying mobile needs to be idle.

To find the percentage of area added for coverage by introducing a carrier or relay, we denote the total number of grids in the cell, given by ' $\mathrm{N}$ '. Out of these grids let ' $\mathrm{n}$ ' have one or more below threshold areas. So initial above threshold areas are N-n. Now out of $n$ grids we need to find the probability of placing a stretched call to determine the increase in coverage. The occurrence of BTAs is given by the GPD. So given the BTAs, the probability of $\mathrm{j}$ stretched calls is given by $P(j \mid n)$. If there is constant number of mobiles in the cell, the number of idle nodes changes after one has been allocated. So this follows hypergeometric distribution given as,

$$
P(j \mid n)=\frac{\left(\begin{array}{l}
n \\
j
\end{array}\right)\left(\begin{array}{l}
N-n \\
n-j
\end{array}\right)}{\left(\begin{array}{l}
N \\
n
\end{array}\right)}
$$

This is the probability of finding a mobile able to carry a call without being replaced. If we can replace the available set of mobiles, then it will follow the Bernoulli distribution. The probability of finding an intermediary for a mobile is,

$$
P=\sum_{n} P(j \mid n) \cdot P_{X}(\theta, \lambda)
$$




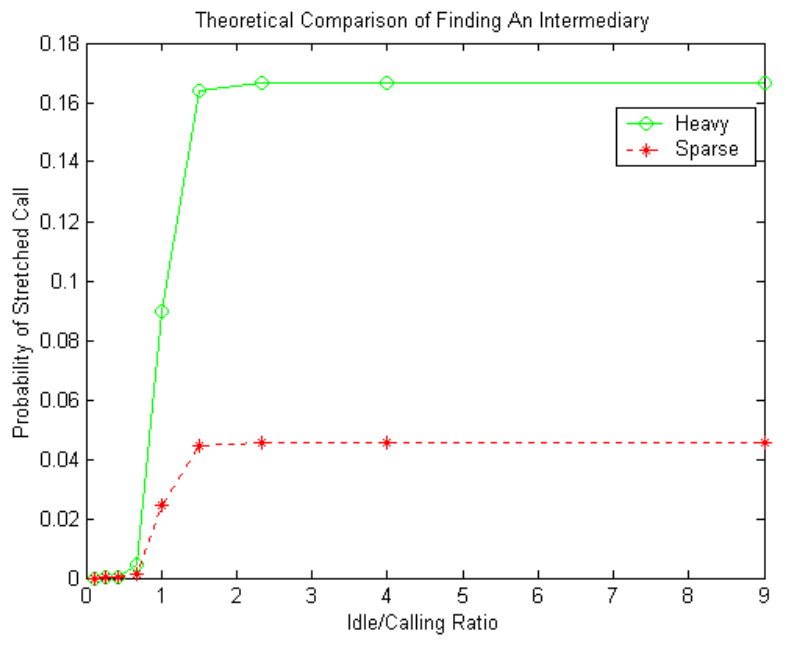

Figure 3. Theoretical probability of finding an intermediary

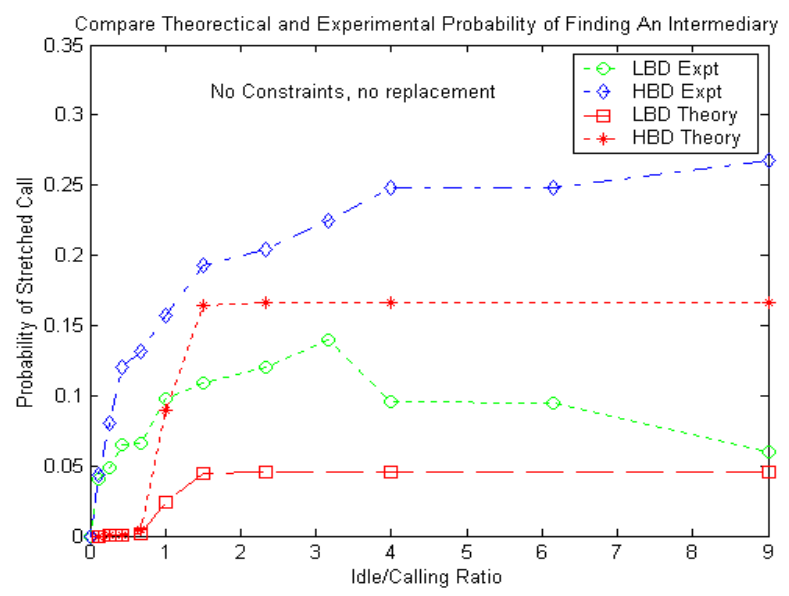

Figure 4. Comparison of theoretical and experimental probability values for stretched call for "No constraint, No replacement" appraoch

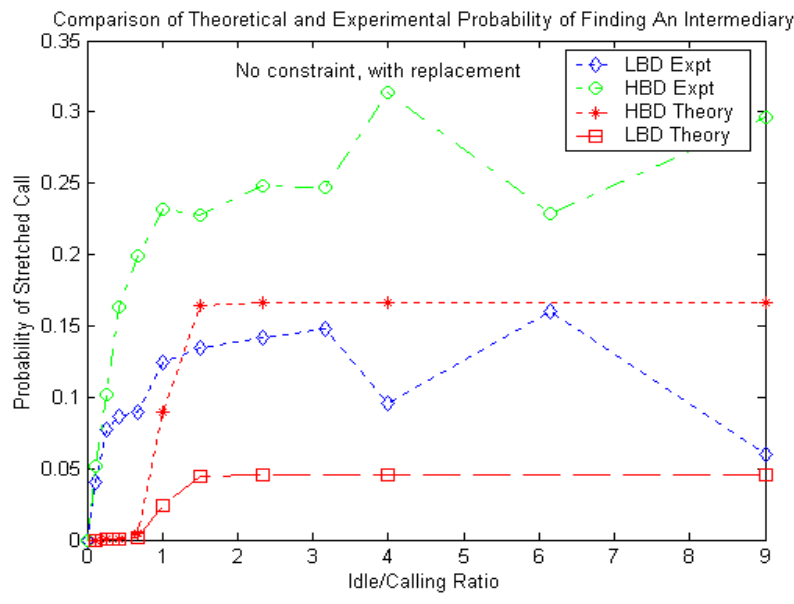

Figure 5. Comparison of theoretical and experimental probability values for stretched call for "No constraint, With replacement" appraoch

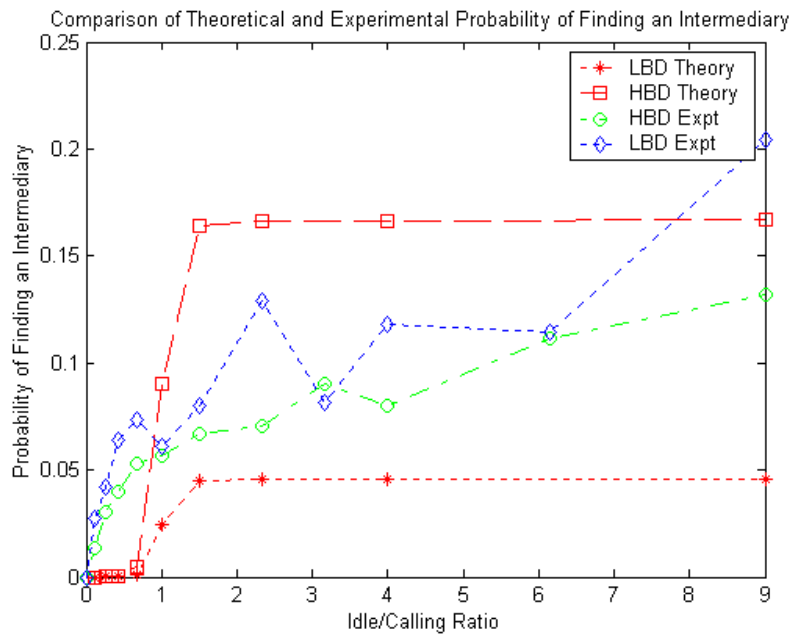

Figure 6. Comparison of theoretical and experimental probability values for stretched call for "With constraint, With replacement" appraoch

We choose heavy building density areas, shown as heavy in Figure 2, with lambda as -0.2 and theta as 0.8 . For low density areas, shown in the same figure as sparse, lambda is taken as -0.1 and theta as 0.2 . In Figure 3 we plot the probability of a mobile using a stretched call due to poor coverage. As expected heavy density areas have a higher probability of using a stretched call. The area of interest is the rising curve for both heavy and sparse areas. This shows that saturation is achieved on reaching a certain idle to calling ratio of mobiles.

\section{SIMULATION AND DISCUSSION}

We ran Monte Carlo simulations with 50 nodes on a $1 \mathrm{~km} \mathrm{x}$ $1 \mathrm{~km}$ cellular area with the base station at the center. The path loss exponent was kept at 2 for distances less than threshold distance and 4 for beyond the threshold distance, which was taken at $300 \mathrm{~m}$ [3]. Both high building density cell (heavy, HBD) and low building density cell (sparse, LBD) are considered. The building density is distributed using Generalized Poisson distribution. The range of building density varies from $10-20 \%$ in sparse cell and $10-40 \%$ in heavy cell [3]. The intermediary for a stretched call is chosen if it requires less transmit power compared to a direct call to base station. In some simulations we utilize a constraint that makes sure that the carrying mobile itself doesn't fall into a below threshold area. The approaches considered for allocation of an intermediary are,

1. "No constraint and No replacement" approach - The approach of "No constraint" means that any available mobile is considered for carrying, even if it is lying in a below threshold area. This is possible if the intermediary has higher battery power; say dedicated intermediaries or the main objective is to come out of dead spot. "No replacement" means that the same pool of mobiles is kept throughout the simulation. The initial available set of idle to calling mobiles is considered as the idle to calling ratio.

2. "No constraint and With Replacement" - In this approach the above constraint is not considered. "With 


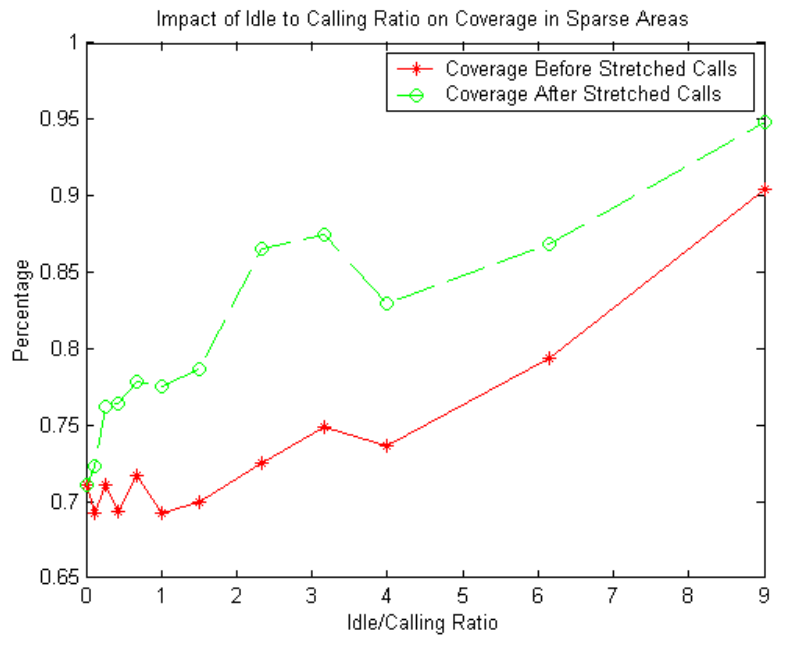

Figure 7. Improvement in reliability of coverage in sparse areas

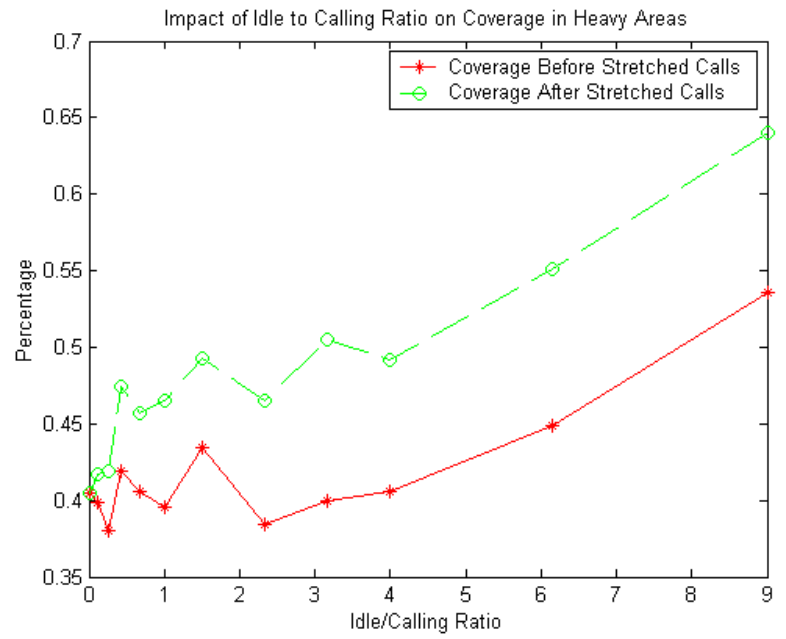

Figure 8. Improvement in reliability of coverage in heavy areas

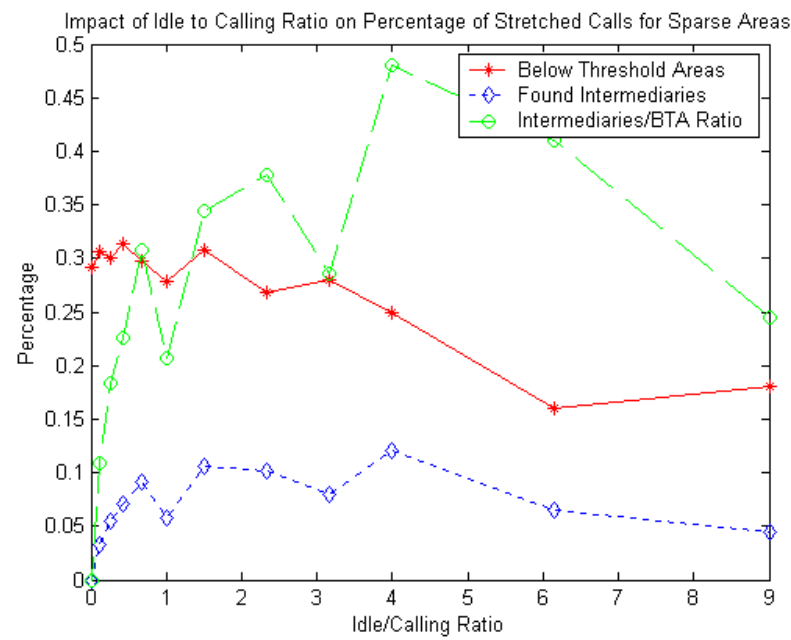

Figure 9. Impact of Idle to Calling Ratio of Mobiles for sparse area

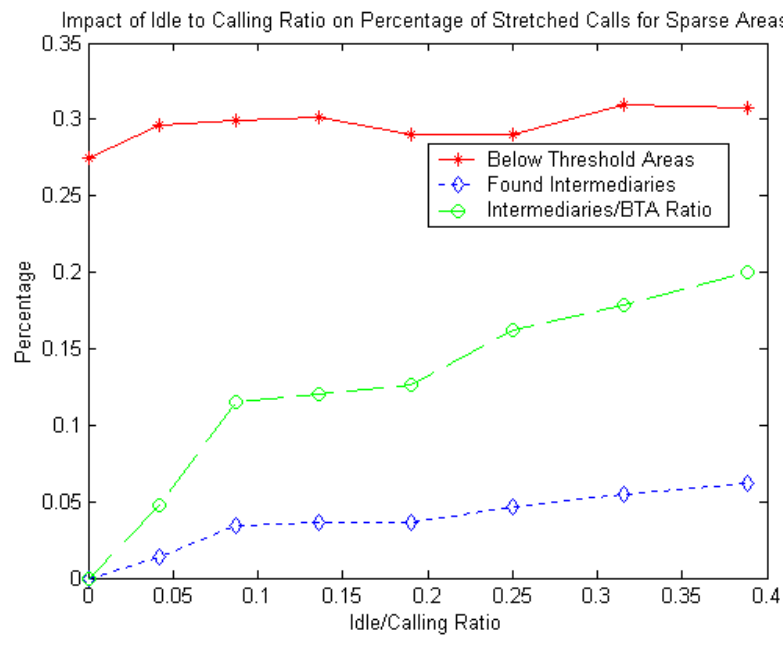

Figure 10. Lower range of Idle to Calling Ratio and its impact on probability of stretched call for sparse area

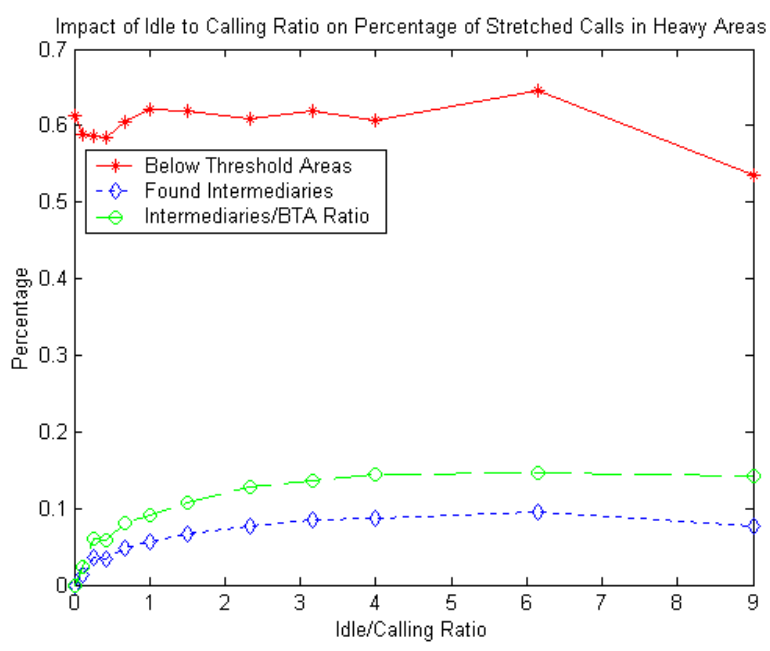

Figure 11. Impact of Idle to Calling Ratio of Mobiles for heavy area

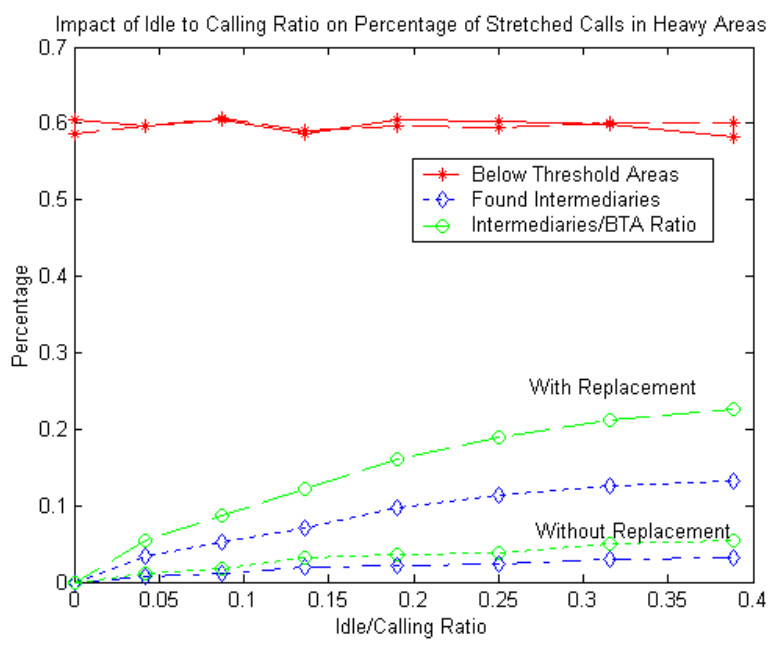

Figure 12. Lower range of Idle to Calling Ratio and its impact on probability of stretched call for heavy area 
replacement" means that new idle mobiles are added as intermediaries are allocated. So the idle to calling ratio is kept constant throughout the simulation.

3. "With constraint and With Replacement" - This approach considers both the above constraints and replaces the idle mobiles allocated as intermediaries. This is the most realistic approach as we don't want the intermediary to fall in a below threshold area.

Figures 4-6 show the comparison of our theoretical model with simulation for these three approaches. For Approaches 1 and 2, shown in Figures 4 and 5, we see that the theoretical model underestimates the probability of a stretched call whereas the model is fairly accurate for Approach 3 (Figure 6). We have to note that the simulations used a decision making process based on power for choosing an intermediary.

The simulation results given below for HBD and LBD are only for "with constraint and with replacement" approach for reasons of space. Figures 7 and 8 show the improvement in coverage with the addition of stretched model, for sparse and heavy areas respectively. For the sparse case, using the stretched call improves coverage from $4-14 \%$. For the heavy areas, improvement is from $5-10 \%$.

In Figures 9, 10, 11, 12 we plot:

- Percentage of mobiles that lie in BTAs ("Below Threshold Areas")

- Percentage of calling mobiles lying in BTAs that use intermediaries ("Found Intermediaries")

- A ratio of the above two ("Intermediaries/BTA ratio")

Figures 9 and 11 correspond to sparse areas and heavy areas respectively. As expected the percentage of mobiles in BTAs is greater for heavy areas. The interesting scenario in Figure 9 occurs at low values of idle to calling node ratio. Here, before the ratio hits one, there is a steady increase in the number of intermediaries that were found. This also corresponds to a higher number of calling nodes. We plot this behavior in detail as shown in Figure 10. This holds lot of promise as the more crowded a cell would be, a little relaxing of call density can bring about a greater probability of finding a carrier. Figure 12 shows this behavior for heavy area. In this, both "with replacement" and "without replacement" approaches along with the constraint are shown for comparison. There are improvements shown by "with replacement" but they are not as high as the without constraint approach would have given. The reason is that the new mobile being added could itself lie in a below threshold area. In heavy areas this is quite probable.

\section{CONCLUSION}

We have shown a significant improvement in the reliability of coverage by using a stretched call, in which an intermediary is used to carry calls from mobiles located in BTAs. We considered heavy and sparse areas and computed the probability of finding an intermediary when the mobile is at a below threshold area. Our results indicate that with an increase in idle to calling ratio, the coverage increases, as does the percentage of stretched calls.

\section{REFERENCES}

[1] Theodore S. Rappaport, "Wireless Communications", Prentice Hall Communications, NJ, 1996.

[2] Mathias Coinchon, Ari-Pekka Salovaara, Jean-Frederic Wagen, "The Impact of Radio Propagation Predictions on Urban UMTS Planning”, International Zurich Seminar on Broadband Communications, 2001.

[3] Jhong Sam Lee and Leonard E. Miller, "CDMA Systems Engineering Handbook", Artech House Publications, Boston, 1998.

[4] A. Neskovic, N. Neskovic and D. Paunovic, "Macrocell Electric Field Strength Prediction Based Upon Artificial Neural Networks", IEEE Journal on Selected Areas in Communications, Vol. 20, No.6, pp1170$1177,2002$.

[5] Venugopal V. Veeravalli and Andrew Sendonaris, "The CoverageCapacity Tradeoff in Cellular CDMA Systems", IEE Trans. Veh. Tech., Vol. 48, No. 5, pp. 1443-1450, 1999.

[6] S. Lakkavalli, A. Negi and S. Singh, "Stretchable Architectures for Next Generation Cellular Networks", Proceedings of the International Symposium on Advanced Radio Technologies, pp 59-65, March 4-7, 2003.

[7] George N. Aggelou and Rahim Tafazolli, "On the Relaying Capacity of Next Generation GSM Cellular Networks”, IEEE Personal Comm., pp. 40-47, Feb 2001.

[8] M. Bronzel, W. Rave, P. Herhold, G. Fettweis, "Interference Reduction in Single Hop Relay Networks", 11th Virginia Tech Symposium on Wireless Personal Communications, 2001.

[9] Brendan C. Jones and David J. Skellern, "Designing the Ubiquitous Microcellular Network", Workshop on Applications of Radio Science, 1995.

[10] P.C. Consul, "Generalized Poisson Distributions, Properties and Distributions”, Marcel Dekker, Inc., New York, 1989. 